\title{
CONSENSUS CONFERENCE REPORT
}

\section{MECHANICAL CARDIAC SUPPORT 2000: CURRENT APPLICATIONS AND FUTURE TRIAL DESIGN}

June 15-16, 2000

Bethesda, Maryland

Lynne Warner Stevenson, MD, FACC, Conference Co-Chair

Robert L. Kormos, MD, Conference Co-Chair

\section{STEERING COMMITTEE MEMBERS}

ROBERT C. BOURGE, MD, FACC ANNETINE GELIJNS, PhD BARTLEY P. GRIFFITH, MD RAY E. HERSHBERGER, MD, FACC SHARON HUNT, MD, FACC JAMES KIRKLIN, MD, FACC LESLIE W. MILLER, MD, FACC
WALTER E. PAE, JR, MD, FACC GEORGE PANTALOS, PhD D. GLENN PENNINGTON, MD, FACC ERIC A. ROSE, MD, FACC JOHN T. WATSON, PhD JAMES T. WILLERSON, MD, FACC JAMES B. YOUNG, MD, FACC

\section{WRITING GROUP PARTICIPANTS}

MARK L. BARR, MD

MARIA ROSA COSTANZO, MD, FACC PATRICE DESVIGNE-NICKENS, MD ARTHUR MICHAEL FELDMAN, MD, PhD, FACC

O. HOWARD (BUD) FRAZIER, MD, FACC

LAWRENCE FRIEDMAN, MD

J. DONALD HILL, MD, FACC

MARVIN A. KONSTAM, MD, FACC

PATRICK McGUANE McCARTHY, MD
ROBERT E. MICHLER, MD, FACC

MEHMET C. OZ, MD, FACC

BRUCE R. ROSENGARD, MD

WOLF SAPIRSTEIN, MD

RHONA SHANKER

CRAIG R. SMITH, MD

RANDALL C. STARLING, MD, MPH, FACC

DAVID O. TAYLOR, MD, FACC

ALISON WICHMAN, MD

\section{INVITED REPRESENTATIVES OF INDUSTRY}

DALLAS W. ANDERSON

KEN CHARHUT

LAURA DAMME, RN, MPH

MICHAEL R. DEVRIES

LORENZO DICARLO, MD, FACC

DAVID J. FARRAR, PhD

LEONARD A. R. GOLDING, MD, FACC

J Thorac Cardiovasc Surg 2001;121:418-24

Copyright $\odot 2001$ by the American College of Cardiology

Reprinted with permission of the American College of Cardiology. 12/1/113857

doi: $10.1067 / \mathrm{mtc} .2001 .113857$
STEVEN A. KOLENIK

TIM KRAUSKOPF

DOUGLAS McNAIR, MD, PhD

TOFY MUSSIVAND, PhD

CHISATO NOJIRI, MD, PhD

GEORGE P. NOON, MD

STEVEN J. PHILLIPS, MD, FACC

PEER M. PORTNER, PhD, FACC

ERIC SCHORSCH

JOSEPH J. SCHWOEBEL, MBA

WINSTON UMEMURA

ROBERT L. WHALEN, PhD

HELENE ZINTAK, PA 


\section{Sponsoring organizations}

The conference was conducted with financial and other support from the following organizations: American College of Cardiology, American Heart Association, International Society for Heart and Lung Transplantation, American Society of Transplantation, Heart Failure Society of America, The American Association for Thoracic Surgery, the Society of Thoracic Surgeons, and the American Society of Transplant Surgeons.* Additionally, participants included members of these agencies: Food and Drug Administration, National Institutes of Health, and the American Society for Artificial Internal Organs.

\section{Impact statement}

Heart failure presents an increasing public health burden of morbidity and mortality even as the mortality from coronary artery disease and hypertension is decreasing. While effective pharmacologic therapies have improved outcomes for mild-moderate heart failure, the impact of newer therapies and mechanical circulatory support for advanced heart failure has not yet been realized. Implantable devices have been shown to be safe and effective as bridges to cardiac transplantation, but further work is needed to establish the role of mechanical support for myocardial recovery and for long-term support. This conference was held to assess current mechanical support applications and future trial designs for investigation affecting this public health issue.

The participants concluded that important differences between devices and drugs may warrant novel study designs characterized by innovation and flexibility. While the randomized clinical trial remains the most powerful tool for unambiguous comparison of interventions, variations may include timed graduation from control to investigational therapies, assignment influenced by patient risk or patient preferences and criteria for an optional crossover to compassionate device use. A major impact would result from a national outcomes database for advanced heart failure that identifies high-risk populations with the greatest potential for benefit from newer therapies and thus facilitates the design of devices and device trials. A separate registry with industry of outcomes after device placement would help to identify "breakthrough" device therapies and facilitate the refinement and acceptance of this new technology. As represented in this

\footnotetext{
"The recommendations set forth in this report are those of the conference participants and do not necessarily reflect the official position of the American College of Cardiology. The full text document will be published in the Journal of the American College of Cardiology and the Journal of Heart and Lung Transplantation, and the executive summary will be published in Circulation and the Journal of Thoracic and Cardiovascular Surgery. This document is available on the World Wide Web site of the American College of Cardiology (www.acc.org). Reprints of this document are available for $\$ 5.00$ each by calling $800-253-4636$ (U.S. only) or by writing the Resource Center, American College of Cardiology, 9111 Old Georgetown Road, Bethesda, Maryland 20814.
}

conference, progress in mechanical circulatory support will be accelerated by the continued coordination of scientists, engineers, industry, clinical investigators and regulatory and payment agencies in prospective partnership.

\section{Introduction}

Over the past five years, mechanical circulatory support devices have evolved from the earlier investigational stages to become standard therapy for bridging to transplantation, in some cases extending beyond original indications. As the first randomized controlled trial of mechanical circulatory support, the Randomized Evaluation of Mechanical Assistance in the Treatment of Congestive Heart Failure (REMATCH) trial began in 1998 and has undergone regular protocol modifications resulting from experiences gained with the patient population and the devices themselves. In 1999, an expert review panel for the National Heart, Lung, and Blood Institute (NHLBI) recommended continued support for the development of total artificial heart programs. Refinement of currently available left ventricular (LV) devices continues steadily, and many new types of support devices are in or approaching clinical trials. Ethical and practical issues have emerged regarding the design and funding of these future clinical trials. Challenges for optimal application are being compounded as the separation between indications for recovery, bridge to transplantation and permanent use is becoming less distinct.

As in the original conference on trial design for mechanical circulatory support led by Pae in 1995, the goals of investigators, governmental agencies and industry remain the establishment of clinical trials that are "scientifically sound, clinically meaningful and achievable in a finite time frame at reasonable expense." With the rapid increase in experience with populations of advanced heart failure, broader clinical application of available devices and the promise of new technology for future support, members of the steering group for the NHLBI, the Food and Drug Administration (FDA), the American College of Cardiology Committee on End-Stage Heart Failure and the International Society for Heart and Lung Transplantation sought broad representation from professional societies and industry to address the issues involved in trial design for mechanical circulatory support looking ahead from 2000.

The professional societies with significant interest in this field were invited to co-sponsor this conference and to select delegates to participate in the discussion and writing of the draft document. The writing groups established the basis of their conclusions for discussion 
and subsequent revision by all participants during the conference at the Heart House in Bethesda, Maryland, to which representatives of industry were also invited. The published document represents the consensus of the participants, as approved by the Steering Committee, and does not imply formal acceptance by any of the societies represented. New developments will render the specifics of this document obsolete, but it is hoped that the fundamental considerations established here will help to guide trial design and clinical decisions for the near future.

\section{Executive summary}

Present status of devices for heart failure. Current use of mechanical circulatory support devices is dominated by the indications of post-cardiotomy shock and bridging to cardiac transplantation. In the U.S., about 6,000 patients a year receive support devices after cardiac surgery, with hospital survival of $20 \%$ to $40 \%$. Sustained improvement of native heart function after support also occurs in $5 \%$ to $15 \%$ of transplant candidates, with greater frequency of recovery in patients with fulminant myocarditis. Bridging to cardiac transplantation occurs in 300 to 400 patients yearly in the U.S., with an overall discharge rate of $50 \%$ to $70 \%$ from device implantation through transplantation.

Limitations in our current conception of device indications need to be recognized. First, the need for biventricular versus univentricular support is difficult to determine. Second, the ultimate utility of a total artificial heart versus ventricular assist device(s) (VAD) has not been established. Third, the intended duration of mechanical support is a moving target. The time and type of device utilization is influenced by external factors such as the time to myocardial recovery, donor organ availability, the potential of outpatient therapy and the unpredictability of adverse events associated with new technology. Thus, even within the field of currently used devices, evolving indications mandate flexible guidelines for utilization.

Development of drugs and surgical devices for advanced heart failure. Observation provided the basis for early therapies of heart failure, many of which have subsequently been abandoned. A systematic approach to testing pharmacologic therapies in heart failure has arisen only within the last 20 years. The basis of evidence supporting the current medical therapy with angiotensin-converting enzyme inhibitors and beta-adrenergic receptor antagonists has arisen from double-blind, randomized controlled trials in hundreds to thousands of patients with mild to moderate heart failure. Except for digoxin, oral inotropic agents have been shown in controlled trials to increase mortality, despite sound theoretical rationale. The template of the double-blind, randomized control trial has emerged as the gold standard for evaluating new pharmacologic therapies. It has not been applied to urgent therapies such as diuretics for relief of pulmonary edema and intravenous inotropic agents for cardiogenic shock (CS), during which placebo therapies might be regarded as unacceptable.

Many surgical approaches have been introduced for heart failure. The coronary artery surgery trial demonstrated benefit in patients with reduced left ventricular ejection fractions (LVEFs) but did not target patients with symptomatic heart failure. Requiring five years to complete enrollment, the trial of revascularization for acute CS demonstrated benefit in patients $<75$ years of age. Revascularization, valve surgery and other remodeling techniques are being employed for some patients with more severe chronic heart failure (HF). The inability to provide comparable placebo therapy, strong patient preferences regarding invasive procedures, and the front-loaded risk of operative procedures have complicated the evaluation of these new approaches.

Fundamental differences between drugs and devices. As therapies for heart failure advance beyond drugs into procedures and devices, fundamental differences emerge in the evaluation of efficacy. By contrast with drug development, progress with devices is more incremental, with experience leading to progressive device modifications. The impact of devices is more transparent, in part because the most obvious risks are frontloaded compared with those from new drugs. It is harder for the effects of devices to be masked or mimicked by the natural history of heart failure. Practical considerations relate to the higher order of magnitude of expense per patient in a trial, which can be prohibitive for companies without major revenue from previous products. The clinically meaningful benefit, however, is projected to be larger than the benefit of new drugs, such that estimated sample sizes are in hundreds rather than thousands of subjects. The experience and skill necessary to achieve optimal outcomes restrict center participation in trials and limit the generalizability of results. A crucial difference between drugs and devices is the inability to blind patients or physicians to therapy, a limitation with both ethical and practical implications for clinical trials.

The sum of evidence guiding therapy with drugs is dominated by evidence from large trials completed prior to drug approval. Once it is approved, it is difficult to identify use and attribute effects of any particular drug because of variable prescription, adherence 


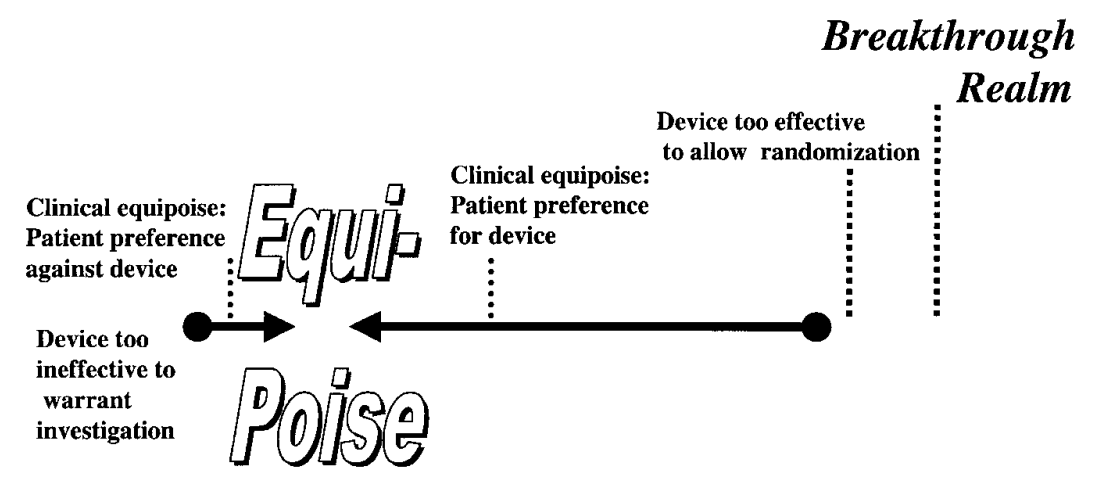

Fig 1. Line depicting the relationship between equipoise and efficacy of a new therapy, as perceived after initial clinical testing. It is possible that the early experience could be so dramatic that both the scientific and regulatory community regard it as a "breakthrough" therapy that should be approved without further investigation for the defined population. Initial experience could also demonstrate sufficient success that the scientific community is convinced of efficacy, while the regulatory agencies require further information. This gap might be bridged by continued clinical investigation at limited sites, with prospective definition of a non-randomized cohort for comparison. In the majority of cases, initial testing does not establish efficacy, and clinical equipoise can be maintained for the performance of randomized controlled trials. It is anticipated that patient preference regarding new therapies will most often lie to the right of clinical equipoise, complicating trials of therapies that cannot be blinded. The asymmetry of the line to the right of equipoise reflects the enthusiasm necessary to drive any therapy through clinical evaluation.

and combination with other medications. For this reason, post-marketing surveillance provides limited information regarding drugs for heart failure, except for non-cardiovascular side effects. By contrast, the very complexity and undisguised impact of devices render their use and outcomes easier to track, as long as appropriate registries are maintained. The cumulative body of evidence guiding the ultimate use of devices may be drawn more from information gained after initial approval.

Target populations and end points for mechanical circulatory support. Target populations for mechanical circulatory support can be defined by the expected natural history of heart failure. Patients with CS have an in-hospital mortality of $>50 \%$ but also carry high risk for patient-related operative complications. Ambulatory patients without resting symptoms on standard oral therapy often survive for two years or longer. Despite various approaches to risk stratification, it remains hard to specify an intermediate-risk population. For patients receiving outpatient intravenous inotropic therapy, the six-month mortality is currently in the range of $50 \%$. However, without objective indications for and restrictions on this therapy, it may encroach on the population with less advanced disease. Another target population might be cardiac transplant patients with triple vessel coronary artery disease
(CAD) and decreased ejection fraction, with $<50 \%$ one-year survival, but mechanical devices in the posttransplant population may be complicated by previous surgery and immunosuppression. The target population for trials should be defined widely to include patients with the best natural history compatible with the degree of certainty that a given device will provide an improvement. This would be greatly facilitated by a multicenter registry of advanced heart failure. After approval, ongoing re-evaluation of a successful device should reflect the observed trend for downshifting risks, in which procedures with proven benefit in a high-risk population become generalized to patients with less risk of post-operative complications but potentially less benefit.

End points for clinical trials will be chosen according to the severity of disease in the population selected. For patients with the most severe disease, early survival will be a fundamental end point. A combination of early survival and functional end points may be most appropriate for trials allowing eventual device placement in patients randomized to medical therapy. As the risk of death becomes imminent, measurements of functional capacity, quality of life and survival adjusted for patient preferences become increasingly relevant. At all levels, measures of efficacy will need to be supplemented by measures of cost-effectiveness. It 
should be emphasized, however, that cost-effectiveness for a successful device is likely to improve after approval, as experience is gained and costs are decreased.

The spectrum including "breakthrough" devices. In the future, initial studies could identify a therapy with such an obvious impact on survival that it would be considered a "breakthrough" for a population with otherwise high early mortality (Fig 1). In retrospect, cardiac transplantation was considered a breakthrough that has been widely accepted without a controlled study. Most new therapies do not enter the breakthrough realm during preliminary testing but fall somewhere else along the spectrum before approval. Outside of breakthroughs, there may be some therapies that are not yet approved but are considered by experienced clinicians to be so effective that waiting for a controlled trial would not be ethical. The best way to bridge this gap to expedite approval from regulatory agencies has not yet been determined for any of the life-threatening diseases. The focus of this conference is not on the approval process but on designing trials of devices for which there is reasonable doubt regarding efficacy. Even for devices in the breakthrough realm for endstage disease, the design of trials would remain relevant for extension to those populations with lesser severity of illness, in whom the benefit of the device could not be assumed.

Trial design for mechanical circulatory support. All new devices are required by the Medical Device Amendments Act to be "safe and effective," as shown through "well-controlled scientific studies" or "valid scientific evidence." Because mechanical circulatory support devices fall into the highest of three risk categories, the sponsor must conduct clinical trials before the FDA grants a pre-marketing approval (PMA) decision. Multiple challenges characterize the performance of these trials for mechanical support devices. Because device innovation, exemplified by left ventricular assist devices (LVADs), is incremental and iterative, it is difficult to determine when a device should come to clinical trial and which aspects of development should be "frozen" while modification continues throughout the investigational and post-marketing stages. There is little precedent for trial design when a high severity of illness limits the duration of observation and humanistic concerns dictate consideration of alternate therapies outside protocol. Other life-threatening illnesses, such as cancer and AIDS, have led to consideration of research designs to minimize ethical conflicts and shorten the PMA processes while shifting more emphasis to rigorous post-marketing studies.
The randomized controlled trial (RCT) remains widely regarded as the most powerful and sensitive tool for comparing therapeutic interventions and the most persuasive force for the acceptance of new technology. Many of the differences between drugs and devices, as detailed in the preceding text, complicate the translation of RCTs from pharmaceutical trials to trials of mechanical support devices.

Ethics of randomized controlled trials for mechanical circulatory support. Special emphasis was placed by this conference on consideration of the ethics of RCTs for mechanical support devices. A fundamental tenet of the ethical RCT is that equipoise exists for the treatment being tested; it would thus not be ethical to do an $\mathrm{RCT}$ of a device already determined from initial testing to be in the breakthrough realm for the population being considered. Theoretical equipoise, in which available data and investigator preference are exactly balanced, may in fact never be located for the individual clinician. Clinical equipoise, in which genuine debate and uncertainty exist among the clinical community, is more feasible and relevant. Although it was initially challenged for the REMATCH trial, the position of equipoise was strengthened by the analysis of pilot data from the pilot trial for REMATCH (PREMATCH), in which no clear survival benefit from the LVAD could be seen at three months.

After randomization has taken place, the patient and his physician are aware of the selected therapy, unlike participation in the placebo arm of a double-blinded drug trial. The combination of life-threatening disease and unblinded therapy raises ethical issues beyond that of physician equipoise at the start of the trial. The visible impact of the device may threaten maintenance of equipoise for investigators following patients during the course of a trial. Responding as individuals to unfiltered information, patients are less likely to be in positions of equipoise even before randomization. Patients consenting to new trials are likely to be already biased toward the procedure and thus may perceive randomization to the control arm as a loss of hope, with potentially deleterious impacts on individual outcomes.

Practical issues of randomized controlled trials for mechanical circulatory support. Patient preference for specific therapies perceived to be life-saving may limit enrollment, particularly when a similar therapy is perceived to be offered by other routes. From a methodologic aspect, randomization does not eliminate evaluation bias when all parties know the treatment received. Patient dissatisfaction regarding treatment choice threatens compliance with follow-up and increases the likelihood of off-protocol therapy that could compro- 
mise the trial results, as was seen in early trials of AZT for AIDS.

The cost of initiating a randomized trial for a new device greatly exceeds that of continuing to report uncontrolled experience. For this effort to be undertaken, the ultimate value in terms of acceptance as an effective device must be consistently endorsed. Financial impediments have profoundly impaired the conduct of clinical trials of devices, for which there have been substantial unreimbursed costs. These disincentives to enrollment increase the duration and overall cost of the study, delaying the time to potential recovery of development costs. Government support for reimbursement of routine Medicare treatment costs and "conditional coverage" of treatment costs in recognized scientifically-designed trials are strongly endorsed by this conference.

Despite a number of obstacles, an RCT of classical design is nearing completion to determine the impact of an implantable mechanical circulatory support device as destination therapy compared with optimal medical therapy. If the REMATCH trial proves a survival benefit for devices in this population, similar devices may be tested against this benchmark. Regardless of the outcome of this trial, both the lessons learned during its conduct and the ultimate results will have a profound influence on the design of future trials.

Modifications of the randomized controlled trial for mechanical cardiac support. It should be recognized that the gold standard methodology for evaluating the impact of a treatment on outcome remains the randomized, double-blinded, placebo-controlled trial. It should also be recognized, however, that surgical interventions in advanced illness may not appropriately lend themselves to all aspects, such as blinding, of this methodological gold standard. With increasing appreciation for the unique aspects of mechanical circulatory support for advanced heart failure, variations in the design of randomized trials merit consideration.

The aspects of randomization and a control arm can be retained in a nonblinded trial with an option to receive active device therapy as "compassionate use" after the achievement of a predefined time or intermediate end points. (Because only the original cohorts would be compared, this does not represent a true crossover design.) This feature may encourage recruitment and retention, while re-aligning incentives for the patient and physician to continue full efforts after randomization to a control arm. Models for randomized trials that allow some degree of patient preference could improve recruitment and patient satisfaction while providing more information on outcomes for patients not desiring device therapy. The degree to which patient preference should influence the choice of therapy remains a major ethical issue for this and other life-threatening conditions. From a more practical standpoint, it is not clear to what extent the advantages of design modifications would outweigh the increase in sample size that would be required.

Comparison of non-randomized cohorts. In the absence of a randomized control group, there are no large historical groups that could be considered for comparison. Contemporary cohort studies offer better information than observational reports without comparison, but they are compromised by a major bias in favor of new treatments. Data provided by a cohort analysis of the bridge-to-transplant experience indicated a major benefit from the device for that indication. While these cohort data were often cited to suggest that a randomized trial of therapy in non-transplant candidates was not ethical, its relevance to this different population was questioned when the small randomized pilot trial indicated no major difference in early outcomes between the device and optimal medical therapy.

Alternatively, to generate prospective control groups, cohortscould be defined by an obligatory control period prior to enrollment that could provide short-to-intermediate-term information, after which, however, subjects entering surgery might be either better or worse than at initial evaluation. Comparison of patients preferring surgery to patients preferring medical therapy would require an extensive adjustment for baseline factors influencing outcome, not all of which can be identified. For non-randomized cohorts, it is not possible to adjust for all of the factors that lead to the provision of a therapy to one patient and not another. A different approach to outcomes adjusted for severity of illness is being investigated for therapy of breast cancer, in which therapy is allocated only to the patients at highest risk, whose outcome is then compared with that projected from a less compromised population on standard therapy, according to a mathematical model. This technique and all of the regression models used to control for cohort differences would require a deeper knowledge of risk profiles and outcomes for advanced heart failure than that which currently exists.

Vital role of registries. The absence of broad-based data and the magnitude of mortality, morbidity and resource utilization argue strongly for the creation of a registry of advanced heart failure. Such a multicenter registry would advance both risk stratification for outcome prediction and the development of a multivariate regression model to help adjust for differences between 
cohorts. Greater confidence in our ability to identify high-risk populations would sharpen trial design and accelerate recognition of devices in the breakthrough realm. Design of RCTs would be streamlined by better selection of target populations and better prediction of event rates.

There is now broad consensus that responsible progress in the field of mechanical circulatory support requires the establishment and maintenance of a mandatory registry that includes all implantable devices, both before and after approval. It should be possible to require specific baseline data collection on patients with mechanical assist devices after device approval if that stipulation is formally linked to the initial approval. By contrast to pharmaceutical therapies, which are easier to study before approval and harder to track afterward, mechanical circulatory support devices may, with appropriate registry documentation, be supported by a weight of evidence distributed differently between pre- and post-approval experiences.

The near future. The lessons learned through the use of current technology have led to formative strategies regarding the timing of implantation, rehabilitative potential and discharge management in patients supported with circulatory assist devices. However, limitations of systems requiring external power sources connected through percutaneous drivelines have led to the development of numerous systems that are as completely implanted in the body as possible. This has resulted in developments along two broad approaches. The first is a refinement of implantable pulsatile systems, including the Abiomed and Penn State/3M total artificial hearts, the Thoratec IVAD, the Novacor II, the World Heart Heartsaver VAD and the Arrow LionHeart VAD. The majority of these systems utilize transcutaneous power transmission and either an integral or component volume compensatory mechanism. A second thrust utilizes a completely new concept of axial flow technology for chronic support and includes the Nimbus/TCI HeartMate II, Intracorporeal Ventricular
Assist System (IVAS), the Jarvik 2000 IVAS and the DeBakey/Micromed IVAS. These systems also depend on transcutaneous power transmission but eliminate the need for volume compensation. The AB-180 Circulatory Support System, the HeartMate III LVAD and the CorAide are devices based on centrifugal principles. In many ways our limited understanding of the impact of this latter group of devices may dictate newer study design principles.

Although there are no specific standards for the preclinical evaluation of newer mechanical circulatory support systems, guidelines do exist. A Preliminary Draft Guidance for Ventricular Assist Devices and Total Artificial Hearts issued by the FDA in December 1987 needs to be updated. The joint paper developed by the American Society for Artificial Internal Organs (ASAIO) and the Society of Thoracic Surgeons (STS) addresses only reliability concerns for long-term devices and does not address emerging technology for which a comprehensive standard with criteria for preclinical testing is still needed. The revision of these guidelines becomes even more important as distinctions between short-, intermediate- and long-term support become increasingly blurred during clinical application. An interdisciplinary effort needs to address the development of a comprehensive standard for the preclinical evaluation of blood pumps, taking into account the uniqueness of each system and its intended use, yet remaining sufficiently flexible to incorporate new clinical experience.

As the field moves ahead, it has become clear that no one trial design or set of standards will be ideal or appropriate for all of these devices, populations and stages of development. This document represents both consensus and controversy from leading scientists, clinical investigators, representatives of industry and regulatory agencies. One of the most important achievements of this conference may be the recognition that the pace of real progress in mechanical circulatory support will be accelerated by ongoing collaboration. 